\title{
SOFT HIGH ENERGY SCATTERING IN NONPERTURBATIVE QCD
}

\author{
E. R. Berger \\ LAPTH, Universite Paris-Sud, Batiment 211, \\ F-91405 Orsay Cedex, France \\ E-mail:berger@th.u-psud.fr
}

\begin{abstract}
In this report diffractive high energy reactions are discussed in a functional integral approach where hadronic amplitudes are calculated from vacuum expectation values of lightlike Wegner-Wilson loops. In the first part we calculate elastic differential cross sections for high energy and small momentum transfer elastic protonproton (pp) scattering which are in reasonable agreement with the experimental data. In the second part we consider exclusive $\pi^{0}$ production in ep-scattering. At high energies photon and odderon exchange contribute to this reaction. We show that odderon exchange leads to a much larger inelastic than elastic $\pi^{0}$ production cross section, dominating the $\gamma$ contribution by orders of magnitude. Observing our process at HERA would establish the soft odderon.
\end{abstract}

\section{Elastic pp scattering}

We will consider here some results of 4 for elastic differential cross sections

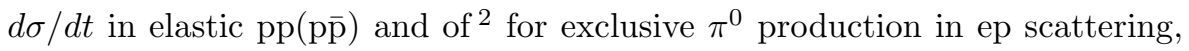
both at high center of mass $(\mathrm{cm})$ energies $\sqrt{s} \gtrsim 20 \mathrm{GeV}$ and low momentum transfer squared $|t| \lesssim \mathrm{O}\left(1 \mathrm{GeV}^{2}\right)$ calculated within a functional approach 3 .

We start with elastic pp scattering. For large energies the amplitude $T_{\mathrm{pp}}\left(T_{\mathrm{p} \overline{\mathrm{p}}}\right)$ is known to be dominated by $C=P=+1$ (pomern $\mathcal{P}$ ) exchange,

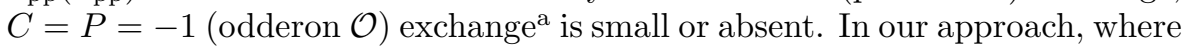
the $\mathrm{p}$ is treated as a wave packet of its constituent quarks, this is achieved by considering the proton to be a quark-diquark (q-qq) system 6 , what we will do in the following. Then the result for $T_{\mathrm{pp}}$ is:

$$
\begin{aligned}
& T_{\mathrm{pp}}=(-2 i s) \int d^{2} b_{T} \exp \left(i \vec{q}_{T} \vec{b}_{T}\right) . \\
& \quad \int d^{2} x_{T} d^{2} y_{T}\left|\Psi^{\mathrm{p}}\left(x_{T}\right)\right|^{2}\left|\Psi^{\mathrm{p}}\left(y_{T}\right)\right|^{2} \widetilde{J}\left(\vec{x}_{T}, \vec{y}_{T}, \vec{b}_{T}\right) ;
\end{aligned}
$$

The scattering amplitude (11) is obtained by first considering the scattering of quarks (antiquarks) on a fixed gluon potential $G$. Travelling through it the quarks (q) and diquarks (qq) pick up non-abelian phase factors. To ensure

a The soft (nonperturbative) odderon is introduced in elastic hadron-hadron scattering as the $C=P=-1$ partner of the pomeron 0 . 

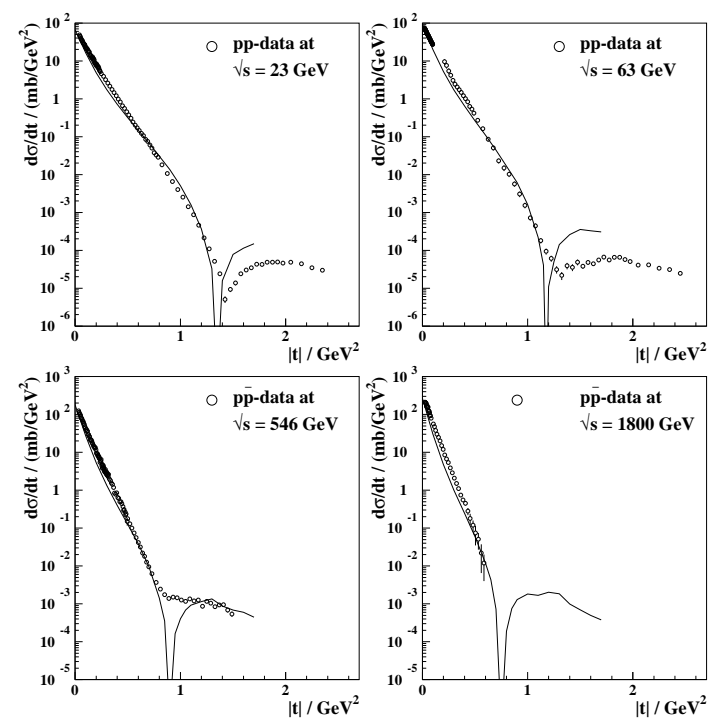

Figure 1: Differential elastic cross sections for c.m. energies $\sqrt{s}=23,63,546$ and $1800 \mathrm{GeV}$.

gauge invariance the phase factors for $\mathrm{q}$ and $\mathrm{qq}$ of the same $\mathrm{p}$ are joined at large positive and negative times, yielding lightlike Wegner-Wilson loops $W_{ \pm}$. The parton scattering amplitude $\tilde{J}$, given by the functional integral over $G$ (indicated with barackets \langle\rangle$_{G}$ ),

$$
\tilde{J}\left(\vec{x}_{T}, \vec{y}_{T}, \vec{b}_{T}\right)=\left\langle W_{+}\left(\frac{1}{2} \vec{b}_{T}, \vec{x}_{T}\right) W_{-}\left(-\frac{1}{2} \vec{b}_{T}, \vec{y}_{T}\right)-1\right\rangle_{G},
$$

is the central object in our approach. The transverse separation between the centres of the loops (impact parameter) is given by $\vec{b}_{T}$. The vectors $\vec{x}_{T}$ and $\vec{y}_{T}$ give the transvers extensions and orientations of the loops. The resulting parton scattering amplitude has to be integrated over $\vec{x}_{T}, \vec{y}_{T}$ with a measure given by the q-qq densities, $\Psi^{\mathrm{p}} \sim \exp \left(-\vec{x}^{2} /\left(4 S_{\mathrm{p}}^{2}\right)\right)$. Here $S_{\mathrm{p}}$ is the p transvers extension parameter. To calculate (1) a Fourier transform over $\vec{b}_{T}$ has to be done finally.

The parton amplitude $\tilde{J}$ is calculated in nonperturbative QCD, using the minkowski version of the model of the stochastic vacuum (MSV). In terms of a matrix cumulant expansion the result is 1 :

$$
\tilde{J}=\left[\frac{2}{3} \cos \left(\frac{1}{3} \chi\right)+\frac{1}{3} \cos \left(\frac{2}{3} \chi\right)-1\right]+i\left[-\frac{2}{3} \sin \left(\frac{1}{3} \chi\right)+\frac{1}{3} \sin \left(\frac{2}{3} \chi\right)\right] .
$$

The real function $\chi$ is calculated from an integrahgver the non-local gluon condensate, for which the MSV makes an ansatz 4 . The parameters that 


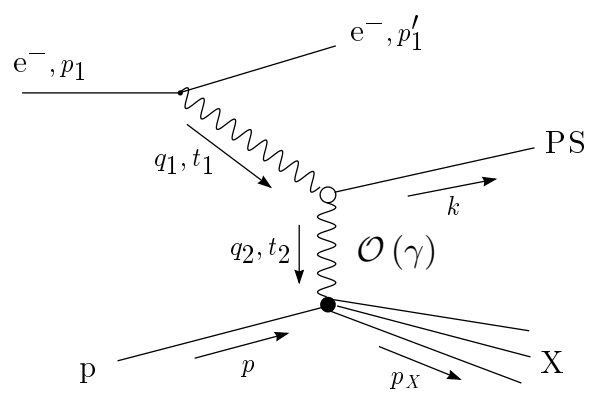

Figure 2: Feynman diagrams for pseudoscalar meson production in $e p$ scattering at high energies with odderon (photon) exchange.

occur are the gluon condensate $G_{2}$, the correlation lenght $a$ and $\kappa$, a parameter measuring the non-abelian content of the condensate. In this way high energy scattering is related to the QCD vacuum parameters.

Now $\chi$ is odd under space inversion or charge conjugation, for example $\chi\left(-\vec{x}_{T}, \vec{y}_{T}, \vec{b}_{T}\right)=-\chi\left(\vec{x}_{T}, \vec{y}_{T}, \vec{b}_{T}\right)$. For this reason $\tilde{J}$ contains $\mathcal{P}$ and $\mathcal{O}$ contributions. But since the q-qq densities in (1) are invariant under space inversion the $\mathcal{O}$ contributions to $T_{\mathrm{pp}}$ average out consistent with experiment?

Leaving $G_{2}, \kappa, a$ and $S_{\mathrm{p}}$ in (11) as free parameters we determine them in high energy scattering, using as input experimental data at $\sqrt{s}=23 \mathrm{GeV}$. We obtain 1 : $G_{2}=(529 \mathrm{MeV})^{4}, \kappa=0.75, a=0.32 \mathrm{fm}$, consistent with existing lattice measurements 0 , and a loop extension parameter $S_{\mathrm{p}}$ in the range of the electromagnetic proton radius, $S_{\mathrm{p}}=0.87 \mathrm{fm}$.

The term of $\chi$ proportional to $\kappa$ results into string-string interaction. This causes $\sigma_{\text {tot }}$ to increase with increasing extension parameter $\boldsymbol{t}_{\boldsymbol{1}}$. Following $\boldsymbol{t}_{\text {we }}$ introduce an energy dependence of (11) by making $S_{p}$ energy dependent, fixed by requiring that our model reproduces the pomeron part of $\sigma_{t o t}$ in the DL parametrisation (The vacuum parameters should not depend on the energy).

In Fig. 1 we show our results for $d \sigma / d t$. A major point is, that our results for $d \sigma / d t$ depend crucially on the string-string interaction, $\kappa \neq 0$, what also implies in terms of the MSV a string tensiop $\rho \neq 0$ and so confinement. A detailed discussion of this point can be found in 1 .

\section{Exclusive $\pi^{0}$ production in ep scattering}

In this section we discuss exclusive $\pi^{0}$ production in high energy ep scattering. The $\pi^{0}$ is produced (see Fig. 2) by $\gamma \mathcal{O}$-fusion and by $\gamma \gamma$-fusion, but can not

\footnotetext{
${ }^{\mathrm{b}} \mathrm{A}$ consequence is, that $T_{\mathrm{pp}}$ and $T_{\mathrm{p} \overline{\mathrm{p}}}$ are equal in the diquark limit. They contain no real part which is due to a second cumulant approximation 1 . As we will see, this results in infinitly deep dips in $d \sigma / d t$
} 


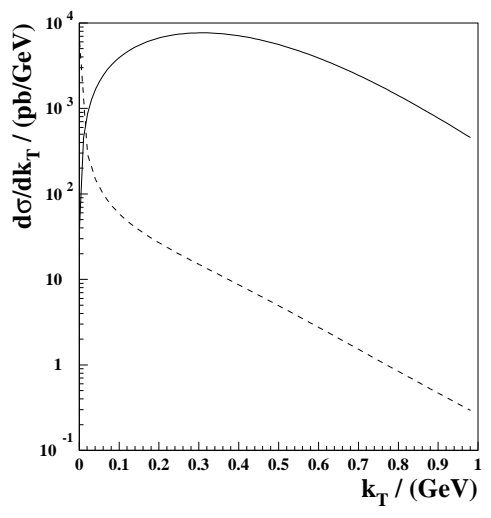

Figure 3: The $k_{T}$ distribution in pion production from the $2 \mathrm{P}$ resonance channels for odderon exchange (solid line) compared to the complete electromagnetic result (dashed line). Interference contributions are not taken into account.

be produced by $\gamma \mathcal{P}$-fusion since the $\pi^{0}$ has positive $C$-parity.

In Fig. 2 $\mathrm{X}$ stands for a proton or resonances or a sum over resonances. Here we treat the very small $Q^{2}:=-q_{1}^{2}$ range where the ep cross section can be calculated by folding the $\gamma$ p cross section with the equivalent photon spectrum of the electron.

To calculate the $\mathcal{O}$ exchange we look at $\gamma p$-scattering in the c.m. system. In the following we discuss the cases, (i) that the proton, stays intact or (ii) gets diffractively exited into the resonances $N(1520)$ with $J^{P}=\frac{3}{2}^{-}$and $N(1535)$ with $J^{P}=\frac{1}{2}^{-}$, described as exited quark-diquark systems.

When considering unpolarised cross sections, summed over both resonances in case (ii), the quark spin degree of freedom becomes irrelevant and the calculation reduces to one where a spinless state stays intact or is exited to a $2 \mathrm{P}$ resonance. The amplitudes are again given by folding $\tilde{J}$ with approperiate wave functions. For (ii) the helicity amplitudes are

$$
\begin{aligned}
& T\left(s_{2}, t_{2}\right)_{\lambda, \lambda_{\gamma}}=-2 i s_{2} \int d^{2} b_{T} e^{i{\overrightarrow{q_{2}}}_{T} \vec{b}_{T}} \hat{J}_{\lambda, \lambda_{\gamma}}(\vec{b}), \\
& \hat{J}(\vec{b})_{\lambda, \lambda_{\gamma}}=\int \frac{d^{2} x_{T}}{4 \pi} \int \frac{d^{2} y_{T}}{4 \pi} \\
& \quad \times \sum_{f, h_{1}, h_{2}} \Psi_{f h_{1} h_{2}}^{* \pi^{0}}\left(\vec{x}_{T}\right) \Psi_{\lambda_{\gamma}, f h_{1} h_{2}}^{\gamma}\left(\vec{x}_{T}\right) \\
& \quad \times \Psi_{\lambda}^{* 2 \mathrm{P}}\left(\vec{y}_{T}\right) \Psi^{p}\left(\vec{y}_{T}\right) \tilde{J}\left(\vec{x}_{T}, \vec{y}_{T}, \vec{b}_{T}\right) .
\end{aligned}
$$

Here $\lambda_{\gamma}(\lambda)$ is the helicity and $\Psi^{\gamma}\left(\Psi^{2 \mathrm{P}}\right)$ the wave function of the photon $(2 \mathrm{P}$ state). Now, when the proton stays intact there occurs in (位) the quarkdiquark density $\left(\Psi_{\lambda}^{2 \mathrm{P}} \rightarrow \Psi^{\mathrm{P}}\right)$. But, since this density is symmetric under a 
parity transformation whereas the odderon coupling changes sign, there is a cancelation when we integrate over all angles. On the other hand when the proton gets exited to a negative parity state like the resonances $N(1520)$ and $N(1535)$ there is no cancellation (the overlap is odd ynder parity) and the odderon couples to the nucleon without any restriction 2. Thus in our model the odderon couples only if breakup of the proton occurs.

The photoproduction cross section calculated from (14) is independent of $s_{2}$ and so the EPA conversion to electroproduction can be achieved by simply multiplying it with a constant $c_{\mathrm{EPA}}=0.0136$.

In the following we consider only ep scattering. An experimentally prefered observable is the $k_{T}$ spectrum, the transverse momentum distribution of the $\pi^{0}$ with respect to the beam direction. This is displayed in Fig. 3. We also show there the distribution of the pion's $k_{T}$ for $\gamma$ exchange summed over the elastic and all inelastic channels (with invariant mass $M_{X} \leq 2 \mathrm{GeV}$ ). As we can see $\gamma$ exchange is larger than $\mathcal{O}$ exchange only for very small $k_{T}$. For $k_{T} \gtrsim 0.1 \mathrm{GeV}$ the $\mathcal{O}$ exchange dominates by orders of magnitude.

\section{Conclusions}

The functional integral approach gives a reasonable description of soft elastic pp scattering at high energies, relating it to fundamental parameters of the QCD vacuum. The values determined from high energy scattering compare well with lattice results. In $\pi^{0}$ production for the $\mathrm{p}$ breakup into negative parity hadronic final states, a large odderon cross section arises which should be observable at HERA. This would establish the soft odderon as an exchangeobject in high energy scattering on an equal footing with the soft pomeron.

\section{References}

1. E. R. Berger, O. Nachtmann, Eur. Phys. J. C7, 459 (1999)

2. E. R. Berger, A. Donnachie, H. G. Dosch, W. Kilian, O. Nachtmann, M. Rueter, Eur. Phys. J. C9, 491 (1999);

3. O. Nachtmann, Ann. Phys. 209 (1991) 436.

4. H. G. Dosch, E. Ferreira, A. Krämer, Phys. Rev. D50 (1994) 1992.

5. L. Lukaszuk, B. Nicolescu, Nuov. Cim. Lett. 8, 405 (1973); D. Joynson, E. Leader, C. Lopez, B. Nicolescu, Nuov. Cim. A 30, 345 (1975)

6. M. Rueter, H. G. Dosch, Phys. Lett. B 380, 177 (1996)

7. E. Meggiolaro, Phys. Lett. B451, (1999) 414

8. M. Rueter, H.G. Dosch, O. Nachtmann, Phys. Rev. D59, 014018, 1999 